\title{
Features for calculating beams with a thin transverse corrugated web plate considering bending-twisting forces
}

\author{
Nikolay Tishkov ${ }^{*}$ and Anatoliy Stepanenko \\ Pacific National University, Khabarovsk, 680035, Tihookeanskaya St. 136, Russia
}

\begin{abstract}
The article describes the features of the work of beams with a thin transverse corrugated web plate. Exponential fractional regression is presented, which allows one to estimate the relative height of web plate sections working together with flanges, obtained by the authors based on an analysis of numerical experiments. Based on the features of the work, a method is proposed for describing the stress state of an arbitrary crosssection of an I-beam with a thin transverse corrugated web plate (the profile of the corrugations is triangular, trapezoidal, sinusoidal) bent in the plane of the web plate and compressed in the longitudinal direction, using the theory of thin-web platted elastic rods by Professor V.Z. Vlasov. The calculations are given for determining the bending-twisting forces (local bending moments and bimoments arising from the action of the main forces) in an arbitrary cross section.
\end{abstract}

\section{Introduction}

The corrugation of individual elements of thin sheet structures has long been used in aircraft structures (corrugated web plates of wing spars), ship structures (corrugated bulkheads, corrugated hatches, corrugated panels of the hulls), building structures (columns, beams, arches) and bridge structures (corrugated web plates of spans) .

In the aircraft industry, since the $1950 \mathrm{~s}$, wing spars with corrugated web plates have been actively used. Vivid examples are the Space Shuttle (1979), where spars with trapezoidally corrugated web plates are used, and the Buran space shuttle orbiter (1984), where spars with a corrugated closed corrugation profile are used. In shipbuilding, from the beginning of the 20th century, corrugated bulkheads with rectangular corrugations were first used. Beams with corrugated web plates are widespread in bridge building. In the construction of the bridge, the corrugated web plate was first used in the Cognac bridge, built in France in 1986. The use of corrugated structures in the form of load-bearing elements of buildings first began in the mid-1960s in Sweden and Germany. In Russia, the use of corrugated structures began actively in the 80 s of the last century. The Kazakh branch of TsNIIPSK developed coating designs for industrial buildings, which included beams with a span of $24 \mathrm{~m}$ and girders with a span of $6 \mathrm{~m}$, the elements were designed with welded I-sections with thin transverse corrugated web plates.

* Corresponding author: n.tishkov87@gmail.com 
The founder of experimental and theoretical research in Russia in the field of corrugated structures is V.N. Gornov. In 1936, the author first conducted a full-scale test of welded beams with a thin transverse corrugated web plate, drawing conclusions about the high efficiency of this type of structure [1]. In the USA, the first work was published by A.F. Fraser in 1956 [2], proposing a practical calculation of a beam with several wavy web plates, which were used in the construction of the wing of an aircraft.

At present, the most common corrugated structural element is a welded beam of an I-section with a corrugated web plate, the thin web plate of which is continuously corrugated by open (extending orthogonally to the longitudinal axis of the rod) corrugations of inclined (triangular), wavy, parabolic, trapezoid profiles.

The general view of the corrugated beam considered in the study is shown in Fig. 1.

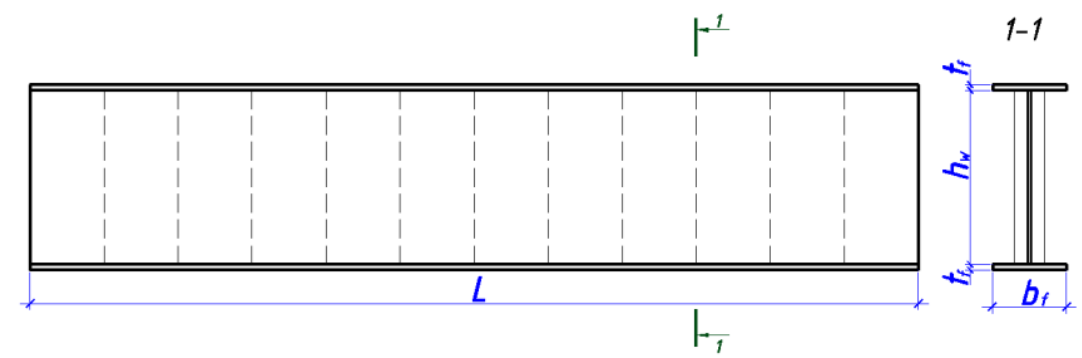

a.
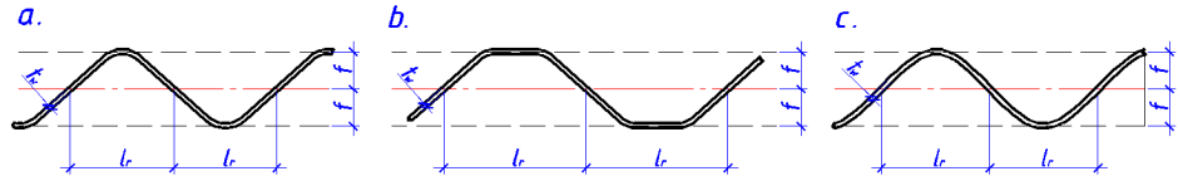

Fig. 1. Linear rod with transversely corrugated web plate

$\mathrm{a}$ - triangular profile of corrugation, $\mathrm{b}$ - trapezoidal profile of corrugation, $\mathrm{c}$ - wavy profile of corrugation

\section{Features of the work of I-beams with a thin transverse corrugated web plate}

The work of I-section beams with a thin cross-corrugated web plate has some differences from the work of classical plane web beams. So the transversely corrugated web plate, at most of its height, behaves like a "bellows" of a harmonica and practically does not take up efforts in the direction across the corrugations, only sections of the web plate work near the flanges, and with sharply decaying diagrams of normal stresses with distance from the flanges. At the same time, the entire transverse force is taken up by the cross-corrugated web plate section, and the distribution of shear stresses along the web plate height is uniform and practically independent of the corrugation profile [1, 3-6].

The work of corrugated beam flanges also has some features. Many researchers, based on the modelling and testing of corrugated web plate beams, concluded that normal stresses in the flanges of beams are not evenly distributed.

In 1971, A.N. Stepanenko [7] at the Khabarovsk Polytechnic Institute conducted a study of beams with a wavy web plate. Studies are devoted to the work of the wavy web plate, the generators of which are directed at different angles to the flange of the beam. Theoretically and experimentally, the author proves that the considered inclined corrugation of the web plates of I-beams does not include the web plate for transverse bending, the web plate only redistributes the forces between the flanges, thereby loading them along the corrugations forming normal stresses. The author describes the stress state by examining a fragment of a 
flange with cross sections in which the web plate adjoins the flange along its axis. Loading a fragment (Fig. 2) along short sides with normal (uniform) stress and shear force along the line of contact with the web plate, which create a linear shear load in the plane of the flanges $t_{p}=\tau_{w} t_{w} \frac{\pi f}{l_{r}} \cos \frac{\pi x}{l_{r}}$ and cause additional forces acting in an arbitrary cross section of the flange $M_{p}=\tau_{w} t_{w} f \frac{l_{r}}{\pi} \cos \frac{\pi x}{l_{r}}$ and $Q_{p}=\tau_{w} t_{w} f \sin \frac{\pi x}{l_{r}}$. These additional efforts load the flanges of a thin-web platted corrugated rod and should be taken into account in engineering calculations.

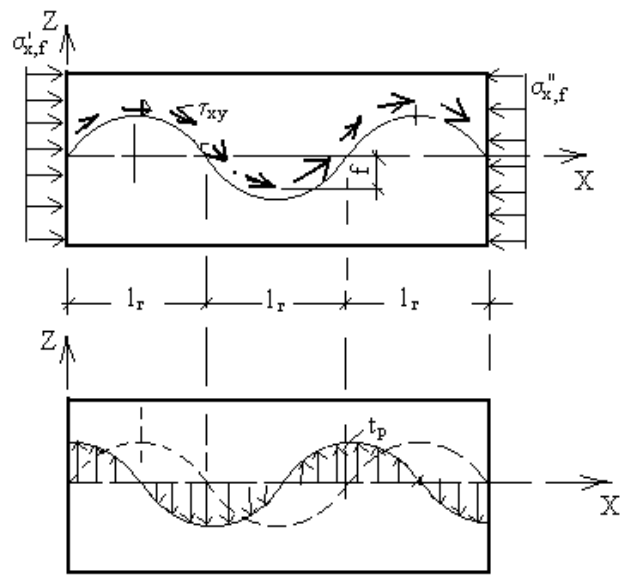

Fig. 2. To the determination of additional effort loads in flanges

Based on the results of studies of beams with corrugated web plates, a calculation method is proposed. Theoretical studies conducted by the author are in good agreement with experimental data.

In 1992, J. Linder [8] conducts a series of large-scale tests of corrugated structures, on the basis of which he concludes that the entire bending moment is taken up by the flanges, and additional normal stresses arise in the flanges. Based on the test results, patterns of the distribution of additional normal stresses in the flanges were obtained. In 1997, the study was continued by R. Aschinger and J. Lindner [9]. The authors proposed to determine the forces in the flanges as for a beam loaded with an additional transverse force Fy and a transverse bending moment $\mathrm{Mz}$ in the plane of the flanges, as shown in Fig. 3.

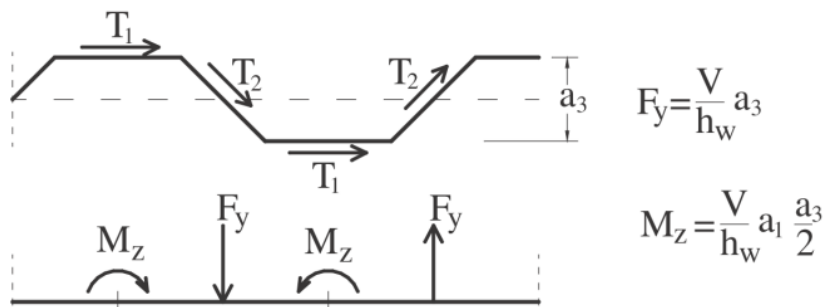

Fig. 3. Theoretical substantiation of additional lateral force and moment in flanges 
The force Fy and My cause lateral bending of the flanges sheets, in connection with which additional normal stresses arise, which have a variable sign.

Significant research in the field of corrugated structures was carried out by H.H. Abbas, R. Sauce and R.G. Driver $[6,10,11]$. The study analysed the work of corrugated beams under load, as well as the distribution of normal stresses in the flanges. Large-scale specimens with a trapezoidal profile of corrugations were tested. According to the test results, a numerical model was developed on the basis of which the work of the elements of the corrugated beam was analysed. The authors justify additional efforts in the flanges by the presence of transverse forces and bending moments acting in the plane of the flanges, similarly to R. Aschinger and J. Lindner [9]. At the same time, the authors note another type of additional effort caused by periodic torsion of the beam relative to the centre of gravity of the flanges (Fig. 4).
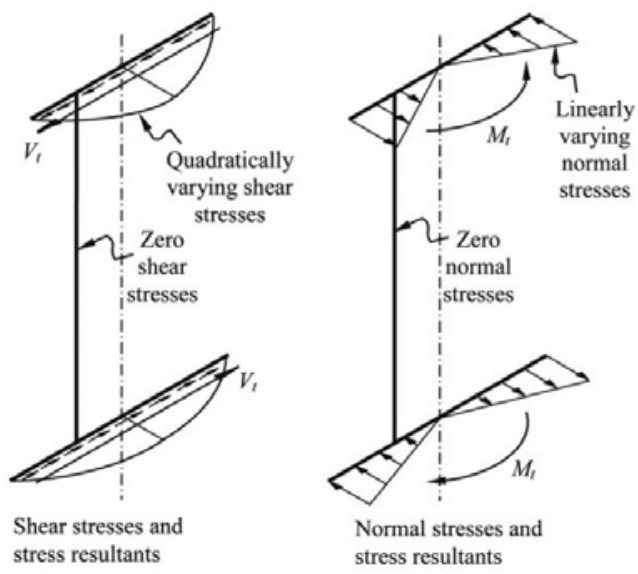

Fig. 4. Additional stresses in the flanges caused by torsion of the beam relative to the centre of gravity of the flanges

The theoretical studies conducted by the authors are in good agreement with the experimental data obtained in the course of laboratory tests and a numerical experiment. It was also noted that additional stresses can reach significant values and should be taken into account in the calculations.

\section{Additional bending-twisting forces and stresses in a composite beam of an I-section with a thin cross-corrugated web plate}

In 2001, A.N. Stepanenko [12] considers I-shaped elements with a wavy web plate as thin-web platted spatial rods using the theory of thin-web platted elastic rods V.Z. Vlasov and describes their actual stress-strain state. In the course of theoretical and experimental studies, the author found that the periodic displacement of the web plate from the axis of the rod leads to the displacement of the centres of gravity and the centres of bending of the sections, leading to the appearance in the section of additional forces in the form of bending (from the action of the main axial force in the rod), bending torque, bimoment and the moment of pure torsion (from the action of the main bending moment in the rod and cutting force). The marginal normal stresses in the core flanges from these additional efforts can reach up to $40 \%$ of the value of the main values. The theoretical calculations 
of the author are consistent with the experimental studies and confirm the possibility of using the results in practical calculations.

Moreover, in the work [12], when determining local (additional) forces in the flanges of steel beams with a thin transverse corrugated web plate caused by the displacement of the centres of gravity of their reduced sections from the axis of the flanges, half the web plate section was taken into account in the joint work with each flange In reality, together with each flange, in most cases, only a small part of the web plate $(0.1-0.3$ of its height) works with longitudinal efforts, with sharply decaying diagrams of normal stresses with distance from the flanges (Fig. 5.)

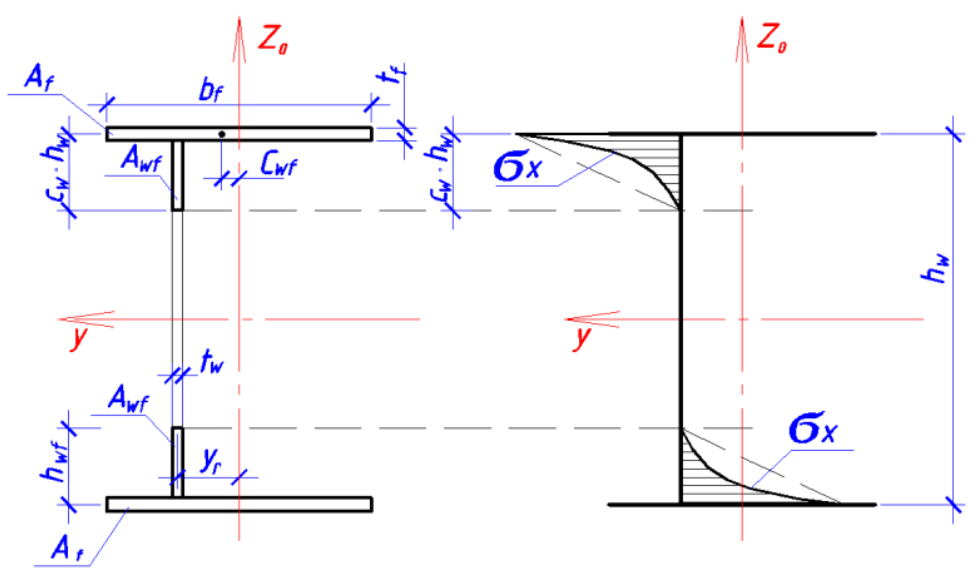

Fig. 5. Normal stress distribution in the web plate of a corrugated beam

Therefore, in the reduced sectional area of the flanges with a certain margin $A_{w f}=0,5 c_{w} h_{w} t_{w}$, only these parts of the web plate of magnitude can be included. In this case, the shift of the centre of gravity of the reduced section of the flange from the axis of the beam in the plane of the flange will be equal to: $c=\frac{A_{w f}}{A_{w f}+A_{f}} y_{r}=k_{c} y_{r}$,

where $y_{r}$ is the magnitude of the displacement of the web plate from the axis of the beam $y_{r}=f \phi(x)$;

$f$ - height of the corrugation profile;

$\phi(x)$ - a continuous function that describes the shape of the profile of the corrugations along the axis of the beam.

$\phi(x)=\sin \frac{\pi x}{l_{r}}-$ a function for the sinusoidal profile of the corrugations or close in shape [12];

$\phi(x)=\frac{4}{\pi \alpha} \sum_{k=0}^{\infty} \frac{\sin ((2 k+1) \alpha) \sin \left(\frac{(2 k+1) \pi x}{l_{r}}\right)}{(2 \mathrm{k}+1)^{2}}-$ general function for describing the triangular and trapezoidal corrugation profiles [13], where the coefficient $\alpha$ determines the shape of the corrugation profile.

The relative height of the sections of a thin web plate with continuous transverse corrugations of a triangular profile working in conjunction with flanges can be 
approximately determined by fractional power regression $c_{w} \cong 0,44 \frac{l_{r}^{0,78} f^{0,11}}{h_{w}^{0,85} t_{w}^{0,03} b_{f}^{0,01}} \leq 0,5$ obtained by the authors by a numerical experiment [14] conducted for cross sections passing along the vertices of the corrugations of the web plate, uniformly loaded along the entire length.

\subsection{Additional efforts from the bending moment}

To determine the additional efforts from the bending moment, it can be assumed that the bending moment is taken up only by flanges in which longitudinal forces opposite in sign occur $N_{1}=M_{y} / h$ (Fig. 6 ).
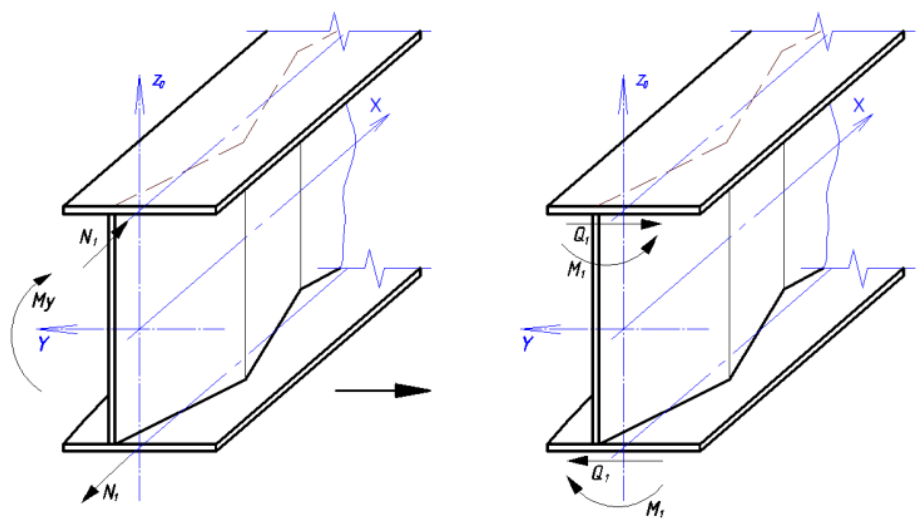

Fig. 6. Additional efforts in the flanges of an I-beam with a thin transverse corrugated web plate from bending moment

The centre of gravity of the reduced section is shifted from the axis of the rod, in connection with which there are local (additional) oppositely directed moments in the flanges $M_{1}=\frac{M_{y}}{h} c$, which, taking into account the profile of the corrugations, can be determined:

- for the sinusoidal profile of the corrugations $M_{1}=\frac{M_{y}}{h} f k_{c} \sin \frac{\pi x}{l_{r}}$;

- for triangular and trapezoidal profiles of corrugations $M_{1}=\frac{M_{y}}{h} k_{c} \frac{4 f}{\pi \alpha} F_{2}(x)$, where $\mathrm{F}_{2}(x)=\sum_{k=0}^{\infty} \frac{\sin ((2 k+1) \alpha) \sin \left(\frac{(2 k+1) \pi x}{l_{r}}\right)}{(2 \mathrm{k}+1)^{2}}$.

Local moments varying along the length of the rod cause local (additional) transverse forces in the section flanges, which are opposite in direction $Q_{1}=\left(M_{1}\right)^{\prime}$ :

- for the sinusoidal profile of the corrugations $Q_{1}=\frac{M_{y}}{h} f k_{c} \frac{\pi}{l_{r}} \cos \frac{\pi x}{l_{r}}$; 
- for triangular and trapezoidal profiles of corrugations $Q_{1}=\frac{M_{y}}{h} k_{c} \frac{4 f}{l_{r} \alpha} F_{1}(x)$, where $F_{1}(x)=\sum_{k=0}^{\infty} \frac{\sin ((2 k+1) \alpha) \cos \left(\frac{(2 k+1) \pi x}{l_{r}}\right)}{2 k+1}$.

Local oppositely directed moments in the section form a bending-twisting moment $B_{\omega 1}=M_{1} h$, and a pair of transverse forces (also oppositely directed) bending-twisting $M_{\omega 1}=Q_{1} h$.

\subsection{Additional efforts from cutting force}

Assuming that the shear force along the height of the web plate is distributed evenly and equally $q_{c}=Q_{z} / h$ (Fig. 7).
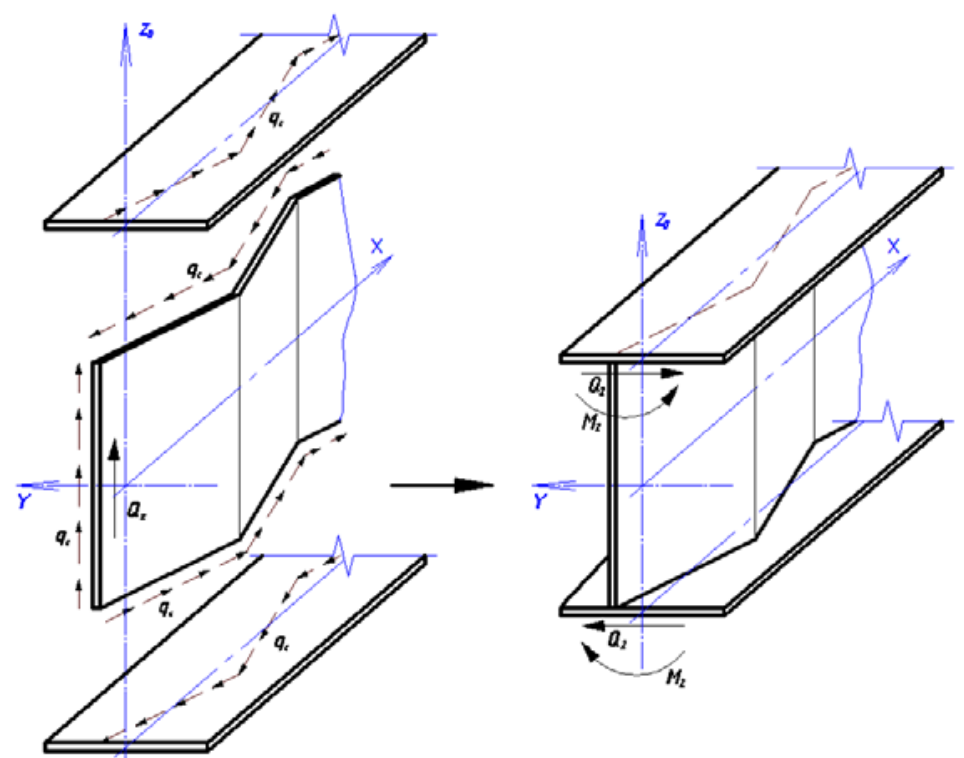

Fig. 7. Additional forces in flanges of an I-beam with a thin transverse corrugated web plate from the cutting force

Separating the flange flanges of length $L$ from the web plate and loading them along the line of contact with the web plate with shear forces equal (due to the law of pairwise action of shear stresses) to the linear shear force in the cross section of the web plate, we can determine the projection of the shear force acting on the flange onto its cross section and horizontal supporting reaction of the flange at the beginning of the rod with the hinged support of the latter. These forces cause shear and bending of the flanges in their planes:

- for a sinusoidal profile of corrugations $Q_{2}=\frac{Q_{z} f l_{r}}{h \pi L}\left(1-\cos \frac{\pi L}{l_{r}}-\frac{\pi L}{l_{r}} \sin \frac{\pi x}{l_{r}}\right)$; $M_{2}=\frac{Q_{z} f l_{r}}{h \pi}\left[\frac{x}{L}\left(1-\cos \frac{\pi L}{l_{r}}\right)-1+\cos \frac{\pi x}{l_{r}}\right] ;$ 
- for triangular and trapezoidal profiles of corrugations $Q_{2}=\frac{Q_{z}}{h} \frac{4 f l_{r}}{\pi^{2} L \alpha}\left[F_{3}-F_{3}(L)-\frac{\pi L}{l_{r}} F_{2}(x)\right], M_{2}=\frac{Q_{z}}{h} \frac{4 f l_{r}}{\pi^{2} \alpha}\left[\frac{x}{L}\left(F_{3}-F_{3}(L)\right)-F_{3}+F_{3}(x)\right]$, where $F_{3}=\sum_{k=0}^{\infty} \frac{\sin ((2 k+1) \alpha)}{(2 k+1)^{3}}$

$F_{3}(\mathrm{~L})=\sum_{k=0}^{\infty} \frac{\sin ((2 k+1) \alpha) \cos \left(\frac{(2 k+1) \pi L}{l_{r}}\right)}{(2 k+1)^{3}}$.

These forces, oppositely directed in different flanges, also form, respectively, bendingtwisting and bimoment in the cross section of the considered rod: $M_{\omega 2}=Q_{2} h$; $B_{\omega 2}=M_{2} h$.

\subsection{Additional effort from longitudinal force}

In an I-beam with a thin transverse corrugated web plate, the longitudinal force is taken up only by flanges (Fig. 8).
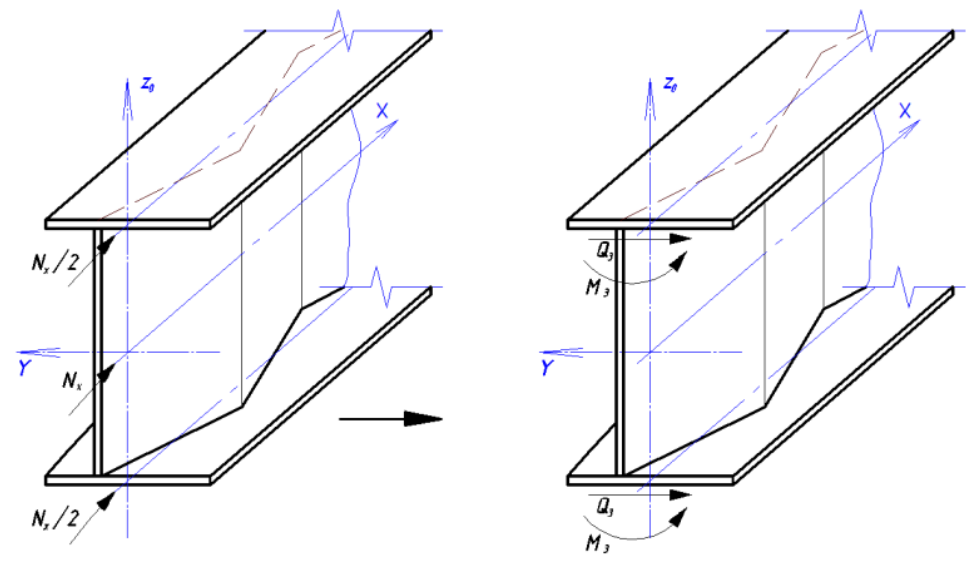

Fig. 8. Additional forces in flanges of an I-beam with a thin transverse corrugated web plate from longitudinal force

When the main axial (compressive) force acts in the rod structures in its flanges, local bending forces directed in one direction arise that do not cause bending torsion:

- for a sinusoidal profile of corrugations $M_{3}=\frac{N_{x}}{2} f k_{c} \sin \frac{\pi x}{l_{r}} ; Q_{3}=\frac{N_{x}}{2} f k_{c} \frac{\pi}{l_{r}} \cos \frac{\pi x}{l_{r}}$;

- for triangular and trapezoidal corrugation profiles $M_{3}=\frac{N_{x}}{2} k_{c} \frac{4 f}{\pi \alpha} F_{2}(x)$, $Q_{3}=\frac{N_{x}}{2} k_{c} \frac{4 f}{\alpha l_{r}} F_{1}(x)$. 


\subsection{Stresses from additional forces in the cross section}

Stresses at the cross-sectional points from additional bending-twisting forces, in accordance with the theory of calculation of thin-web platted rods, are determined by the formulas: $\sigma_{\omega}=\frac{B_{\omega} \omega}{J_{\omega}^{x}} ; \tau_{\omega}=\frac{M_{\omega} S_{\omega}}{J_{\omega}^{x} \cdot t}$,

where $B_{\omega}, M_{\omega}$ are the bending-twisting forces in the section (respectively, the moment of bending and bending-twisting);

$B_{\omega}=B_{\omega 1}+B_{\omega 2} ; M_{\omega}=M_{\omega 1}+M_{\omega 2} ;$

$t$ - the thickness of the element at the cross-sectional point for which the voltage is determined;

$\omega$ - sectorial coordinate for the considered section point;

$S_{\omega}-$ sectorial static moment of the section point.

Additional stresses at the cross-sectional points of the flanges of a centrally compressed (extended) I-beam with a corrugated web plate from local bending forces $\left(M_{3}, Q_{3}\right)$ are determined by the formulas:

$\sigma_{M}= \pm \frac{\left(M_{3}-\Delta M\right) y}{J_{n}} ; \tau_{Q}= \pm \frac{\left(Q_{3}-\Delta Q\right) S_{z}}{J_{n} t_{f}}$

where $J_{n}=\frac{J_{z o}}{2}=\frac{b^{3} t_{f}}{12}$ is the moment of inertia of the flange relative to the $z$ axis;

$y$ - the distance from the considered point of the section of the flange to the $z$ axis;

$S_{z}$ - static moment relative to the $z$ axis, part of the section cut off at a point.

\section{Conclusions}

Based on the statements of the fundamental theory of thin-web platted elastic rods prof. B.Z. Vlasov developed the theoretical principles for calculating I-beams with a thin transverse corrugated web plate with a continuous profile of corrugations, which allow us to describe the actual stress state of an arbitrary cross section. It has been established that the periodic displacement of the centers of gravity and bending of cross sections in the studied structures leads to the appearance of local additional bending-twisting forces from the action of the main forces (axial, transverse, bending), the normal stresses of which at the extreme points of the flanges can reach up to $20 \%$ of the main stresses, and tangents up to $5 \%$.

\section{References}

1. A.V. Gornov, Design and standard, 4, 25-28 (1937)

2. A.F. Fraiser, Experimental investigation of the strength of multiweb beams with corrugated webs, Washington (1956)

3. Yu.S. Maksimov, G.M. Ostrikov, V.V. Dolinsky, Structural mechanics and structural analysis, 1, 68-70 (1983)

4. C.L. Chan, Y.A. Khalid, B.B. Sahari, A.M.S. Hamouda, J. Constr. Steel Res., 58, 3911406 (2002)

5. M. Elgaaly, A. Seshadri, Adv. Eng. Softw., 29, 195-208 (1998) 
6. H.H. Abbas, R. Sauce, R.G. Driver, J. Eng. Mech., 132, 806-814 (2006)

7. A.N. Stepanenko, Construction mechanic and building structures, 133-142 (1971)

8. J. Lindner, Stahlbau, 61, 311-318 (1992)

9. R. Aschinger, J. Lindner, Stahlbau, 66, 136-142 (1997)

10. H.H. Abbas, R. Sauce, R.G. Driver, J. Struct. Eng., 133, 347-355 (2007)

11. H.H. Abbas, R. Sauce, R.G. Driver, Eng. Struct., 29, 2816-2824 (2007)

12. A.N. Stepanenko, Strength and stability of designs from a tee with a wavy web plate, Khabarovsk (2001)

13. P.I. Egorov, Industrial and civil engineering, 10, 34-35 (2007)

14. A.N. Stepanenko, N.L. Tishkov, Bulletin of the ESSU, 4, 41-45 (2016) 